\title{
The electroweak Hamiltonian in the gradient flow
}

\section{formalism}

\author{
Robert Harlander $^{a}$ and Fabian Lange ${ }^{b, c, *}$ \\ ${ }^{a}$ TTK, RWTH Aachen University, \\ 52056 Aachen, Germany \\ ${ }^{b}$ Institut für Theoretische Teilchenphysik, Karlsruhe Institute of Technology (KIT), \\ Wolfgang-Gaede Straße 1, 76128 Karlsruhe, Germany \\ ${ }^{c}$ Institut für Astroteilchenphysik, Karlsruhe Institute of Technology (KIT), \\ Hermann-von-Helmholtz-Platz 1, 76344 Eggenstein-Leopoldshafen, Germany \\ E-mail: harlander@physik.rwth-aachen.de, fabian.lange@kit.edu
}

Over the last decade the gradient flow formalism has become an important tool for lattice simulations of Quantum Chromodynamics. It offers remarkable renormalization properties which pave the way for cross-fertilization between perturbative and lattice calculations. In this contribution we report on the construction of the flowed operator product expansion for the current-current operators of the electroweak Hamiltonian at NNLO QCD. This allows for simpler transformations between lattice and perturbative schemes and might reduce the uncertainties of theoretical predictions for low-energy flavor observables.

*** The European Physical Society Conference on High Energy Physics (EPS-HEP2021), ***

*** 26-30 July $2021 * * *$

*** Online conference, jointly organized by Universität Hamburg and the research center DESY ***

${ }^{*}$ Speaker 


\section{Introduction}

Flavor observables play an important role in the search for new physics beyond the Standard Model (SM). Theoretical predictions of such flavor observables are usually calculated in the effective theory of weak interactions described by an effective Hamiltonian [1]. Typically, the Wilson coefficients are derived from a perturbative matching calculation to the SM (or a particular model for new physics). Depending on the process, their SM expressions are known through nextto-leading order (NLO) or next-to-next-to-leading order (NNLO), see e.g. Refs. [1-5]. On the other hand, the evaluation of matrix elements of the higher-dimensional composite operators requires non-perturbative treatment as provided by lattice QCD, for example. Matching the renormalization conditions of these two factors is an important source of uncertainties for theoretical predictions in flavor physics [6].

The gradient flow formalism (GFF) [7] provides an elegant solution to this problem. It can be viewed as a regularization scheme which applies to lattice as well as perturbative calculations. Composite operators are finite after renormalization of the QCD parameters and the involved fields, without the need for additional operator renormalization [8]. This implies that there is no mixing among these operators under the renormalization group.

In this contribution we consider the current-current operators. Through a suitable basis transformation our results also apply to $|\Delta F|=2$ transitions such as $B \bar{B}$ or $K \bar{K}$ mixing. We introduce the relevant operators in Section 2 and their flowed counterparts in Section 3. In Section 4 we then derive the flowed operator product expansion (OPE) [9-11] for the electroweak Hamiltonian through NNLO. We conclude in Section 5.

Through NLO this strategy has been performed in Ref. [12], albeit in a different operator basis.

\section{Operator basis}

We schematically write the effective Hamiltonian as

$$
\mathcal{H}_{\mathrm{eff}}=-\frac{4 G_{\mathrm{F}}}{\sqrt{2}} V_{\mathrm{CKM}} \sum_{n} C_{n} O_{n}
$$

where $G_{\mathrm{F}}$ denotes the Fermi constant and $V_{\mathrm{CKM}}$ the relevant elements of the Cabbibo-KobayashiMaskawa (CKM) matrix [1]. The Wilson coefficients $C_{n}$ capture the perturbative effects, whereas matrix elements of the operators $O_{n}$ describe mostly non-perturbative effects.

For the physical current-current operator basis we choose [13]

$$
\begin{aligned}
& O_{1}=-\left(\bar{\psi}_{1, \mathrm{~L}} \gamma_{\mu} T^{a} \psi_{2, \mathrm{~L}}\right)\left(\bar{\psi}_{3, \mathrm{~L}} \gamma_{\mu} T^{a} \psi_{4, \mathrm{~L}}\right), \\
& O_{2}=\left(\bar{\psi}_{1, \mathrm{~L}} \gamma_{\mu} \psi_{2, \mathrm{~L}}\right)\left(\bar{\psi}_{3, \mathrm{~L}} \gamma_{\mu} \psi_{4, \mathrm{~L}}\right),
\end{aligned}
$$

where our convention for the color generators is ${ }^{1}$

$$
\left[T^{a}, T^{b}\right]=f^{a b c} T^{c}, \quad \operatorname{Tr}\left(T^{a} T^{b}\right)=-T_{\mathrm{R}} \delta^{a b}
$$

\footnotetext{
${ }^{1}$ This convention differs from the one of Ref. [13] which is the reason for the sign difference in $O_{1}$ between that paper and Eq. (2).
} 
and we adopt Euclidean space-time. The subscript L denotes the left-handed component of the spinors

$$
\psi_{\mathrm{R} / \mathrm{L}}=P_{ \pm} \psi=\frac{1}{2}\left(1 \pm \gamma_{5}\right) \psi .
$$

The dependence of the fields on the space-time variable $x$ is suppressed here and in what follows. Throughout the calculation we assume the quark flavors $\psi_{1}, \ldots, \psi_{4}$ to be mutually distinct. All penguin operators as well as the chromomagnetic operator do not contribute in this case. By a suitable basis transformation, our results apply also to the cases corresponding to $|\Delta F|=2$ processes, i.e. $\psi_{1}=\psi_{3}$ and $/$ or $\psi_{2}=\psi_{4}$ as long as $\psi_{2} \neq \psi_{1} \neq \psi_{4} \neq \psi_{3} \neq \psi_{2}$.

Working in dimensional regularization with $D=4-2 \epsilon$, one has to introduce so-called evanescent operators which vanish for $D=4$, but mix with the physical operators at higher orders in perturbation theory [14]. However, one can prevent the physical operators from mixing into the evanescent operators by a finite renormalization such that all Green's functions of evanescent operators vanish [14-16]. Our choice for the evanescent operators is the same as in Ref. [13].

\section{Flowed operators}

In the GFF, one defines flowed gluon and quark fields $B_{\mu}^{a}=B_{\mu}^{a}(t)$ and $\chi=\chi(t)$ as solutions of the flow-equations $[7,17]$

$$
\begin{gathered}
\partial_{t} B_{\mu}^{a}=\mathcal{D}_{v}^{a b} G_{v \mu}^{b}+\kappa \mathcal{D}_{\mu}^{a b} \partial_{\nu} B_{\nu}^{b} \\
\partial_{t} \chi=\Delta \chi-\kappa \partial_{\mu} B_{\mu}^{a} T^{a} \chi, \quad \partial_{t} \bar{\chi}=\bar{\chi} \overleftarrow{\Delta}+\kappa \bar{\chi} \partial_{\mu} B_{\mu}^{a} T^{a}
\end{gathered}
$$

with the initial conditions

$$
B_{\mu}^{a}(t=0)=A_{\mu}^{a}, \quad \chi(t=0)=\psi,
$$

where $A_{\mu}^{a}$ and $\psi$ are the regular gluon and quark fields, respectively, and

$$
\begin{gathered}
\mathcal{D}_{\mu}^{a b}=\delta^{a b} \partial_{\mu}-f^{a b c} B_{\mu}^{c}, \quad \Delta=\left(\partial_{\mu}+B_{\mu}^{a} T^{a}\right)\left(\partial_{\mu}+B_{\mu}^{b} T^{b}\right), \\
G_{\mu \nu}^{a}=\partial_{\mu} B_{\nu}^{a}-\partial_{\nu} B_{\mu}^{a}+f^{a b c} B_{\mu}^{b} B_{\nu}^{c} .
\end{gathered}
$$

The parameter $\kappa$ is arbitrary and drops out of physical quantities; we will set $\kappa=1$ in our calculation, because this choice reduces the size of the intermediate algebraic expressions.

Our practical implementation of the GFF in perturbation theory follows the strategy developed in Ref. [8] and further detailed in Ref. [18]. On the one hand, it amounts to generalizing the regular QCD Feynman rules by adding flow-time dependent exponentials to the propagators. The flow equations, Eq. (5), are taken into account with the help of Lagrange multiplier fields which are represented by so-called flow lines in the Feynman diagrams. They couple to the (flowed) quark and gluon fields at flowed vertices which involve integrations over flow-time parameters.

Replacing the regular by flowed quark fields in Eq. (2), one arrives at the flowed physical operators

$$
\begin{aligned}
& \tilde{O}_{1}=-\dot{Z}_{\chi}^{2}\left(\bar{\chi}_{1, \mathrm{~L}} \gamma_{\mu} T^{a} \chi_{2, \mathrm{~L}}\right)\left(\bar{\chi}_{3, \mathrm{~L}} \gamma_{\mu} T^{a} \chi_{4, \mathrm{~L}}\right), \\
& \tilde{O}_{2}=\dot{Z}_{\chi}^{2}\left(\bar{\chi}_{1, \mathrm{~L}} \gamma_{\mu} \chi_{2, \mathrm{~L}}\right)\left(\bar{\chi}_{3, \mathrm{~L}} \gamma_{\mu} \chi_{4, \mathrm{~L}}\right)
\end{aligned}
$$


and analogously for the evanescent operators. The non-minimal renormalization constant $\stackrel{\circ}{\chi}_{\chi}$ for the flowed quark fields $\chi$ is defined by

$$
\begin{aligned}
& \stackrel{\circ}{\chi}_{\chi}=-\frac{2 n_{\mathrm{c}} n_{\mathrm{f}}}{(4 \pi t)^{2}} \cdot \frac{1}{\left.\langle R(t)\rangle\right|_{m=0}},
\end{aligned}
$$

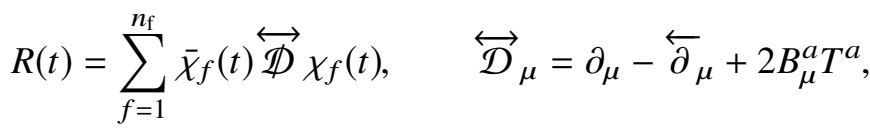

where $\langle\cdot\rangle$ denotes the vacuum expectation value [10], $n_{\mathrm{c}}$ is the number of colors, and $n_{\mathrm{f}}$ the number of quark flavors. $\stackrel{Z}{\chi}_{\chi}$ was computed through second order in the strong coupling constant $\alpha_{\mathrm{s}}$ in Ref. [18].

\section{Flowed operator product expansion}

The flowed operators can be expressed by the small-flow-time expansion [8]

$$
\tilde{O}_{i}(t) \asymp \sum_{j} \zeta_{i j}^{\mathrm{R}}(t) O_{j}^{\mathrm{R}}
$$

where the symbol $\asymp$ signals that this relation holds asymptotically in the limit $t \rightarrow 0$. The mixing matrix $\zeta_{i j}^{\mathrm{R}}(t)$ and the regular operators $O_{j}^{\mathrm{R}}$ are already renormalized here. At the bare level, the mixing from and to the evanescent operators has to be taken into account [14-16]. Only after renormalizing the regular operators can we discard the evanescent operators in Eq. (10).

By inverting Eq. (10) one finds the flowed OPE [9-11]. In the case of the electroweak Hamiltonian of Eq. (1) it reads

$$
\mathcal{H}_{\mathrm{eff}} \asymp-\frac{4 G_{\mathrm{F}}}{\sqrt{2}} V_{\mathrm{CKM}} \sum_{n} \tilde{C}_{n} \tilde{O}_{n},
$$

where the flowed Wilson coefficients are given through

$$
\tilde{C}_{n}=\sum_{m} C_{m}^{\mathrm{R}}\left(\zeta^{\mathrm{R}}\right)_{m n}^{-1}
$$

Since the flowed operators $\tilde{O}_{n}$ do not require renormalization [8], the r.h.s. is scheme independent and one can directly combine the perturbative results for the Wilson coefficients with the matrix elements of the operators obtained by other means, for example a lattice calculation.

By constructing suitable projectors [19, 20] (see also Ref. [21]), we extracted the elements of the mixing matrix $\zeta_{i j}^{\mathrm{R}}(t)$ from Eq. (10). Through NNLO we find

$$
\left(\zeta^{\mathrm{R}}\right)^{-1}=\mathbb{1}+\frac{\alpha_{\mathrm{s}}}{4 \pi}\left(\begin{array}{cc}
16.85 & -3.333 \\
-15 & 14.85
\end{array}\right)+\left(\frac{\alpha_{\mathrm{s}}}{4 \pi}\right)^{2}\left(\begin{array}{cc}
363.5-11.55 n_{\mathrm{f}} & -72.49+2.521 n_{\mathrm{f}} \\
-371.2+11.35 n_{\mathrm{f}} & 311.5-6.934 n_{\mathrm{f}}
\end{array}\right)
$$

where the QCD color factors have been inserted and the renormalization scale has been set to $\mu=\frac{e^{-\gamma_{E} / 2}}{\sqrt{2 t}}$. We obtained these results in general $R_{\xi}$ gauge and with the operator renormalization from the literature, see e.g. Ref. [22]. Both facts provide welcome checks of our calculation. 
For $|\Delta F|=1$ processes the Wilson coefficients $C_{m}^{\mathrm{R}}$ for the SM can be found in Refs. [2,3] through NNLO. Thus, when neglecting penguin contributions, the ingredients for the flowed Wilson coefficients in Eq. (12) are known through NNLO. For $|\Delta F|=2$ processes the two operators in Eq. (2) become related by a Fierz identity and a suitable basis transformation is required. In this case, the SM Wilson coefficient is known through NLO [1], with two contributions for Kaon mixing known through NNLO $[4,5]$.

\section{Conclusions}

In this contribution we constructed the flowed OPE for the current-current operators of the electroweak Hamiltonian through NNLO in QCD. The non-renormalization of the flowed operators removes the need to calculate the scheme transformation between the perturbative Wilson coefficients and the matrix elements from lattice simulations and, thus, could remove one source of uncertainties from theoretical predictions of low-energy flavor observables. Our complete results as well as more details on our calculation and a comparison to the NLO results of of Ref. [12] will be presented elsewhere.

\section{Acknowledgments}

We thank the members of the CERN Theory Department, especially Martin Lüscher, for initiating this project. In addition, we thank him for providing us with his private notes. Furthermore, we thank Ulrich Nierste and Benjamin Summ for stimulating discussions. We acknowledge financial support by the Deutsche Forschungsgemeinschaft (DFG, German Research Foundation) through grant 386986591 and through the Collaborative Research Centre TRR 257 funded through grant 396021762.

\section{References}

[1] G. Buchalla, A.J. Buras and M.E. Lautenbacher, Weak decays beyond leading logarithms, Rev. Mod. Phys. 68 (1996) 1125 [hep-ph/9512380].

[2] C. Bobeth, M. Misiak and J. Urban, Photonic penguins at two loops and $m_{t}$-dependence of $B R\left[B \rightarrow X_{s} l^{+} l^{-}\right]$, Nucl. Phys. B 574 (2000) 291 [hep-ph/9910220].

[3] M. Gorbahn and U. Haisch, Effective Hamiltonian for non-leptonic $|\Delta F|=1$ decays at NNLO in QCD, Nucl. Phys. B 713 (2005) 291 [hep-ph/0411071].

[4] J. Brod and M. Gorbahn, $\epsilon_{K}$ at next-to-next-to-leading order: The charm-top-quark contribution, Phys. Rev. D 82 (2010) 094026 [1007. 0684].

[5] J. Brod and M. Gorbahn, Next-to-Next-to-Leading-Order Charm-Quark Contribution to the CP Violation Parameter $\epsilon_{K}$ and $\Delta M_{K}$, Phys. Rev. Lett. 108 (2012) 121801 [1108. 2036].

[6] Flavour Lattice Averaging Group collaboration, FLAG Review 2019: Flavour Lattice Averaging Group (FLAG), Eur. Phys. J. C 80 (2020) 113 [1902.08191]. 
[7] M. Lüscher, Properties and uses of the Wilson flow in lattice QCD, JHEP 08 (2010) 071 [1006.4518].

[8] M. Lüscher and P. Weisz, Perturbative analysis of the gradient flow in non-abelian gauge theories, JHEP 02 (2011) 051 [1101.0963].

[9] H. Suzuki, Energy-momentum tensor from the Yang-Mills gradient flow, PTEP 2013 (2013) $083 \mathrm{~B} 03$ [1304.0533].

[10] H. Makino and H. Suzuki, Lattice energy-momentum tensor from the Yang-Mills gradient flow-inclusion of fermion fields, PTEP 2014 (2014) 063B02 [1403 . 4772].

[11] C. Monahan and K. Orginos, Locally smeared operator product expansions in scalar field theory, Phys. Rev. D 91 (2015) 074513 [1501.05348].

[12] A. Suzuki, Y. Taniguchi, H. Suzuki and K. Kanaya, Four quark operators for kaon bag parameter with gradient flow, Phys. Rev. D 102 (2020) 034508 [2006.06999].

[13] K. Chetyrkin, M. Misiak and M. Münz, $\|\Delta F\|=1$ nonleptonic effective Hamiltonian in a simpler scheme, Nucl. Phys. B 520 (1998) 279 [hep-ph/9711280].

[14] A.J. Buras and P.H. Weisz, QCD nonleading corrections to weak decays in dimensional regularization and 't Hooft-Veltman schemes, Nucl. Phys. B 333 (1990) 66.

[15] M.J. Dugan and B. Grinstein, On the vanishing of evanescent operators, Phys. Lett. B 256 (1991) 239.

[16] S. Herrlich and U. Nierste, Evanescent operators, scheme dependences and double insertions, Nucl. Phys. B 455 (1995) 39 [hep-ph/9412375].

[17] M. Lüscher, Chiral symmetry and the Yang-Mills gradient flow, JHEP 04 (2013) 123 [1302.5246].

[18] J. Artz, R.V. Harlander, F. Lange, T. Neumann and M. Prausa, Results and techniques for higher order calculations within the gradient-flow formalism, JHEP 06 (2019) 121 [1905.00882].

[19] S.G. Gorishny, S.A. Larin and F.V. Tkachov, The algorithm for OPE coefficient functions in the MS scheme, Phys. Lett. B 124 (1983) 217.

[20] S.G. Gorishny and S.A. Larin, Coefficient functions of asymptotic operator expansions in minimal subtraction scheme, Nucl. Phys. B 283 (1987) 452.

[21] R.V. Harlander, Y. Kluth and F. Lange, The two-loop energy-momentum tensor within the gradient-flow formalism, Eur. Phys. J. C 78 (2018) 944 [1808. 09837].

[22] P. Gambino, M. Gorbahn and U. Haisch, Anomalous dimension matrix for radiative and rare semileptonic B decays up to three loops, Nucl. Phys. B 673 (2003) 238 [hep-ph/0306079]. 